Return to the Manage Active Submissions page at http://spie.org/submissions/tasks.aspx and approve or disapprove this submission. Your manuscript will not be published without this approval. Please contact author_help@spie.org with any questions or concerns.

\title{
A comprehensive set of simulation tools to model and design high performance Type-II InAs/GaSb superlattice infrared detectors
}

\author{
M. Delmas*a , B. L. Liang ${ }^{\mathrm{b}}$, D. L. Huffaker ${ }^{\mathrm{a}, \mathrm{b}}$ \\ ${ }^{a}$ School of Physics and Astronomy, Cardiff University, The Parade, Cardiff, CF243AA, UK \\ ${ }^{\mathrm{b}}$ California NanoSystem Institute, University of California, Los Angeles, CA 90095, USA
}

\begin{abstract}
In this work, the electronic band structure of the InAs/GaSb superlattice (SL) is calculated using a commercial 8-band k.p solver and the electrical performance of longwave $n B p$ device structure evaluated with Atlas from Silvaco software. By taking into account an InSb interface layer and the interface matrix (formulated by P.C. Klisptein), the model can predict the measured energy band gap of different InAs/GaSb SLs having different period composition and thickness (7/4, 10/4, $12 / 4,14 / 4$ and 14/7 SLs) within an error corresponding to the $\pm k_{B} T$ deviation range. The effective mass is then extracted from the electronic band structure calculation and discussed for numerous SL designs. In particular, we compare a 14/7 SL and a 12/2 SL having an energy band gap equal to 0.122 and $0.118 \mathrm{eV}$ at $77 \mathrm{~K}$, respectively. The electron-hole wavefunction overlap for the $12 / 2$ SL has been estimated to be $\sim 74 \%$, which is almost twice the value calculated for the 14/7 SL ( 40\%). This arises from the delocalization of carriers in a thinner SL period also leading to smaller carrier effective masses in the 12/2 SL. The dark-current of a $n B p$ structure has been calculated for both SLs. For the 14/7 SL, the dark-current level has been found to be higher by a factor of over 3 than the Rule07 benchmark, whereas for the 12/2 SL, it is lower by a factor of 0.77 , demonstrating that the SL design can be used to improve the device performances.
\end{abstract}

Keywords: InAs/GaSb superlattice, infrared detector, 8-band k.p model, barrier structure, simulation, long wavelength

\section{INTRODUCTION}

Since the proposal by Smith and Mailhiot in 1987 [1], the Type-II InAs/GaSb superlattice (SL) material has been of great interest as it offers unique properties for infrared detection including a tunable band gap, a high absorption coefficient and low tunneling currents. In recent years, the attention has mainly been focused on developing InAs/GaSb SLs for LongWavelength InfraRed (LWIR) detection, motivated by the growing interest in space applications such as Earth observation, climatology, cold object detection and satellite tracking. Although important results have already been obtained [2-6], the need of simulation tools for performance analysis and device design is increasingly relevant to achieve high performance with low dark-current and high quantum efficiency. Indeed, they are necessary to gain understanding of this SL material system that governs the properties of a detector active region, to properly design emerging barrier structures and to investigate new device concepts.

In this work, we used the 8-band k.p solver implemented in Nextnano ${ }^{3}$ software [7] for the band structure modelling of InAs/GaSb SL and the Atlas software from Silvaco [8] for the device simulation. After describing the simulation methods in section 2, the k.p modelling is calibrated in section 3 by comparing the calculated and measured band gap of SLs having different period composition and thickness. The effective mass is then extracted from the electronic band structure calculation and discussed for different SL designs. In particular, we compare a SL composed of 14 monolayers (MLs) of InAs and $7 \mathrm{MLs}$ of GaSb (14/7 SL) and a 12/2 SL in terms of band structure and material parameters. Finally, the darkcurrent of a $n B p$ structure based on the 14/7 SL and 12/2 SL is evaluated and compared.

*DelmasM@cardiff.ac.uk; phone: (+44) 02922510182 
Return to the Manage Active Submissions page at http://spie.org/submissions/tasks.aspx and approve or disapprove this submission. Your manuscript will not be published without this approval. Please contact author_help@spie.org with any questions or concerns.

\section{METHODS}

\subsection{Electronic band structure calculation using an 8-band k·p envelope-function method}

The 8-band k·p envelope-function method employed for this work is available in Nextnano ${ }^{3}$. The model is described in detail in Ref. [9]. The interface (IF) matrix $H_{I F}$ formulated by P.C Klipstein and implemented in the software framework has been used to model the no atom in common InAs/GaSb SL. It is defined as [10]:

$$
H_{I F}=\sum_{i} \delta\left(z-z_{i}\right)\left[\begin{array}{cccc}
D_{S} & 0 & 0 & \pi_{i} \beta \\
0 & D_{X} & \pi_{i} \alpha & 0 \\
0 & \pi_{i} \alpha & D_{X} & 0 \\
\pi_{i} \beta & 0 & 0 & D_{Z}
\end{array}\right]
$$

where $i$ is the index of the interface at the position $z_{i}$ and $\pi_{i}$ takes a value of -1 or 1 at the InAs-GaSb and GaSb-InAs IFs. The IF parameters $\alpha$ and $\beta$ have been fixed to a value of $0.2 \mathrm{eV}$. $\AA$ [11] whereas the $D$ diagonal IF parameters $\left(D_{S}, D_{X}, D_{Z}\right)$, which are equal to zero in the case of a common atom superlattice, are determined in order to obtain a good agreement between the calculated and measured energy band gap.

To promote strain compensation of the SL layer on GaSb substrate, "InSb-like" IFs at the InAs-GaSb and/or GaSb-InAs IFs are usually formed [12-13] or intentionally grown [14-15] during the SL growth by molecular beam epitaxy (MBE). Homogenous strain on $\mathrm{GaSb}$ is therefore considered for the strain calculation, as well as, an InSb intentional layer at both interfaces. The total thickness of this InSb layer is equal to $10 \%$ of the InAs thickness in one period of SL which is approximately equal to the thickness needed to compensate the tensile strain caused by InAs on GaSb.

The material parameters of InAs, GaSb and InSb binaries used for the k·p band structure calculation are summarized in

Table 1. These parameters are particularly important to calculate the strain and the k.p parameters.

Table 1: Material parameters of InAs, GaSb and InSb binaries used in Nextnano ${ }^{3}$ for the $\mathbf{k} \cdot \mathbf{p}$ band structure calculation. All values can be found in Ref. [11],[16],[17],[18] and [19] which correspond in the table to the superscripts a, b, c, d and e, respectively.

Parameters

Lattice constant $\mathrm{a}_{0}[\AA]$

Energy band gap at $0 \mathrm{~K}[\mathrm{eV}]$

Varshni parameter $\alpha[\mathrm{meV} / \mathrm{K}]$

Varshni parameter $\beta[\mathrm{K}]$

Conduction band mass $\mathrm{m}_{\mathrm{c}}\left(\mathrm{m}_{0}\right)$

Luttinger parameters $\Upsilon_{1}$

Luttinger parameters $\Upsilon_{2}$

Luttinger parameters $\Upsilon_{3}$

Interband matrix element $\mathrm{E}_{\mathrm{p}}[\mathrm{eV}]$

Spin orbit splitting $\Delta_{\mathrm{O}}[\mathrm{eV}]$

Elastic constant $\mathrm{c}_{11}[\mathrm{GPa}]$

Elastic constant $\mathrm{c}_{12}$ [GPa]

Elastic constant $\mathrm{c}_{44}[\mathrm{GPa}]$

Deformation potential $\mathrm{CB} \mathrm{a}_{\mathrm{c}}[\mathrm{eV}]$

Deformation potential $\mathrm{VB} \mathrm{a}_{\mathrm{v}}[\mathrm{eV}]$

Uniaxial deformation potential $b[\mathrm{eV}]$

Uniaxial deformation potential $\mathrm{d}[\mathrm{eV}]$

Valence band offset VBO $[\mathrm{eV}]$

\begin{tabular}{|lll} 
InAs & GaSb & InSb \\
\hline $6.0583^{\mathrm{c}}$ & $6.0959^{\mathrm{c}}$ & $6.4794^{\mathrm{c}}$ \\
$0.410^{\mathrm{c}}$ & $0.812^{\mathrm{c}}$ & $0.240^{\mathrm{c}}$ \\
$0.276^{\mathrm{c}}$ & $0.417^{\mathrm{c}}$ & $0.320^{\mathrm{c}}$ \\
$93^{\mathrm{c}}$ & $140^{\mathrm{c}}$ & $170^{\mathrm{c}}$ \\
$0.022^{\mathrm{a}}$ & $0.042^{\mathrm{a}}$ & $0.135^{\mathrm{c}}$ \\
$19.77^{\mathrm{b}}$ & $11.74^{\mathrm{b}}$ & $34.8^{\mathrm{c}}$ \\
$8.57^{\mathrm{b}}$ & $4.16^{\mathrm{b}}$ & $15.5^{\mathrm{c}}$ \\
$9.43^{\mathrm{b}}$ & $5.28^{\mathrm{b}}$ & $16.5^{\mathrm{c}}$ \\
$22.2^{\mathrm{d}}$ & $22.4^{\mathrm{d}}$ & $23.3^{\mathrm{c}}$ \\
$0.38^{\mathrm{d}}$ & $0.752^{\mathrm{d}}$ & $0.81^{\mathrm{c}}$ \\
$832.9^{\mathrm{c}}$ & $884.2^{\mathrm{c}}$ & $684.7^{\mathrm{c}}$ \\
$452.6^{\mathrm{c}}$ & $402.6^{\mathrm{c}}$ & $373.5^{\mathrm{c}}$ \\
$395.9^{\mathrm{c}}$ & $432.2^{\mathrm{c}}$ & $311.1^{\mathrm{c}}$ \\
$-6.66^{\mathrm{e}}$ & $-9.33^{\mathrm{e}}$ & $-6.94^{\mathrm{c}}$ \\
$-1^{\mathrm{e}}$ & $-1.32^{\mathrm{e}}$ & $-0.36^{\mathrm{c}}$ \\
$-1.8^{\mathrm{c}}$ & $-2^{\mathrm{c}}$ & $-2^{\mathrm{c}}$ \\
$-3.6^{\mathrm{c}}$ & $-4.7^{\mathrm{c}}$ & $-4.7^{\mathrm{c}}$ \\
$-0.56^{\mathrm{c}}$ & $0^{\mathrm{c}}$ & $0.03^{\mathrm{c}}$
\end{tabular}

\subsection{Device simulation}

The electrical characteristic, i.e. dark-current density versus voltage (J-V), of InAs/GaSb SL detectors has been simulated using Atlas software which is a commercial physically-based device simulator. It has already been employed to analyze 


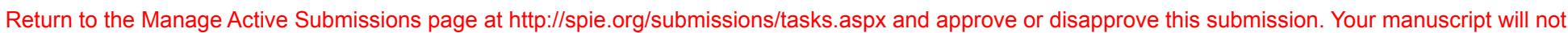
be published without this approval. Please contact author_help@spie.org with any questions or concerns.

performances of III-V infrared detectors [20-22]. Recently, it has been used as a tool to design barrier devices based on SL as reported in Ref. [23] where the contact, barrier and absorber layers of such a structure are optimized in terms of composition, thickness and doping concentration and type.

For J-V simulation, the different components of the dark-current that have been considered are presented in detail in Ref. [22]. The diffusion current is calculated using the drift-diffusion model. The generation-recombination current is described by the Shockley-Read-Hall recombination rate in which a field effect enhancement factor is included to take into account the trap-assisted tunneling contribution. This factor depends mainly on the energy range where the tunneling can occur, the electric field within the device and the tunneling mass $m_{t}$. Finally, the band-to-band tunneling current is calculated using a non-local model which considers the spatial variation of energy bands and assumes that the generationrecombination of opposite carrier types is not spatially coincident, contrary to a local model that calculates the generationrecombination rate at each grid point solely on the electric field value local to this point.

In Atlas, the InAs/GaSb SL material is defined as a bulk material instead of a periodic pile of thin layers. The material parameters used as input for the device simulation are extracted from the electronic band structure calculation, calculated using analytical equations or calculated as a weighted average of InAs and GaSb bulk values. Bulk-based models are in fact used, in particular for the calculation of the intrinsic carrier concentration $n_{i}$ which depends on the effective density of states in the conduction and valence band, and therefore on the electron and hole effective mass. We thus assume that the transport properties of SLs can reasonably be described by defining a bulk material instead. This assumption is particularly true for thin SL periods where the carriers tend to be delocalized and minibands relatively large.

The device structure used in section 3.3 is the same $n B p$ structure as the one presented in Ref. [23]. It consists of a 140 nm n-type $\left(1 \times 10^{16} \mathrm{~cm}^{-3}\right)$ contact layer, a $200 \mathrm{~nm}$ p-type $\left(5 \times 10^{15} \mathrm{~cm}^{-3}\right)$ barrier layer and a $4 \mu \mathrm{m} \mathrm{p}$-type $\left(1 \times 10^{16} \mathrm{~cm}^{-3}\right)$ active region. The contact and absorber layers are composed of the same LWIR InAs/GaSb SL. The barrier layer is considered to have no conduction band offset with the absorber allowing transport of minority electrons and a sufficiently large valence band offset to block the majority holes to reach the contact. The electric field is confined in the high band-gap barrier layer so that the generation-current is suppressed in the absorber. The $n B p$ device is therefore diffusion-limited at low reverse bias (as long as the electric field is confined in the barrier layer) and at any given temperature with a lower dark-current level than the equivalent pin photodiode.

\section{RESULTS AND DISCUSSION}

\subsection{Energy band gap}

The energy band gap extracted from the electronic band structure calculated with the 8-band k-p envelope-function model has been compared to the experimental band gap measured by photoluminescence (PL) at a temperature of $77 \mathrm{~K}$ for different MWIR and LWIR samples grown by MBE. In Table 2, the cut-off wavelength at the PL peak position measured for SLs having different period thickness and composition, i.e. different ratio $\mathrm{R}$ of InAs and GaSb layer thicknesses, is reported. The SL period total thickness and the lattice mismatch $(\Delta \mathrm{a} / \mathrm{a})$ between the SL epitaxial layer and GaSb substrate extracted from high-resolution x-ray diffraction (XRD) spectra are also summarized in this table.

Table 2: SL period thickness extracted from the XRD spectrum along with the lattice mismatch $(\Delta \mathrm{a} / \mathrm{a})$ between the SL epitaxial layer and GaSb substrate for different grown SLs and cut-off wavelength at the PL peak position measured at a temperature of $77 \mathrm{~K}$.

\begin{tabular}{cccc}
\hline $\begin{array}{c}\text { InAs/GaSb SL } \\
(\mathrm{MLs})\end{array}$ & $\begin{array}{c}\text { Period thickness } \\
(\mathrm{nm})\end{array}$ & $\begin{array}{c}\Delta \mathrm{a} / \mathrm{a} \\
(\%)\end{array}$ & $\begin{array}{c}\text { PL peak position } \\
\text { at } 77 \mathrm{~K}(\mu \mathrm{m})\end{array}$ \\
\hline $7 / 4$ & 3.54 & $\sim 0$ & 5.15 \\
\hline $10 / 4$ & 4.51 & $\sim 0.149$ & 8 \\
\hline $12 / 4$ & 5.17 & $\sim 0.06$ & 9.7 \\
\hline $14 / 4$ & 5.75 & $\sim 0$ & 11.3 \\
\hline $14 / 7$ & 6.76 & $\sim 0$ & 10 \\
\hline
\end{tabular}


Return to the Manage Active Submissions page at http://spie.org/submissions/tasks.aspx and approve or disapprove this submission. Your manuscript will not be published without this approval. Please contact author_help@spie.org with any questions or concerns.

In Figure 1, the calculated cut-off wavelength as a function of the measured cut-off wavelength is represented along with the ideal prediction line for which the calculated cut-off wavelength is equal to the measured cut-off wavelength. The $\pm k_{B} T$ deviation (with $k_{B}$ the Boltzmann constant and $T$ the temperature) in the predicted cut-off is also represented. Note that the $D$ diagonal IF parameters $\left(D_{S}, D_{X}, D_{Z}\right)$ of Eq. (1) used are equal to $(0.8,0.3,-0.3)$.

From Figure 1, we observe a good agreement between the calculated and the measured cut-off wavelength for all different SLs with an error in the $\pm k_{B} T$ deviation range, apart from the SL composed of 10 MLs of InAs and 4 MLs of GaSb (10/4 $\mathrm{SL})$. This can be explained by the fact that in our simulation we assume a SL layer lattice matched on GaSb by considering an InSb intentional layer at both IFs. However, from Table 2 it appears that there is large lattice mismatch between the $10 / 4 \mathrm{SL}$ epitaxial layer and the GaSb substrate of about $\Delta \mathrm{a} / \mathrm{a} \sim 0.149 \%$. This compressive strain indicates that there is too much of InSb within the period and that the InAs tensile strain has been over-compensated. In addition, a slight change in the growth rate during the MBE growth can lead to a slight change in composition and thickness of the SL period compared to the targeted layer thicknesses which we do not take into consideration in our simulation. For comparison, the cut-off wavelength calculated without taking into account the interface matrix and without considering either the $H_{I F}$ or the InSb layer at the IFs are also plotted in Figure 1. We can see that if both the $H_{I F}$ and the InSb IF layers are not considered, the model cannot predict the measured cut-off wavelength and under estimates it. This result demonstrates the importance of the interface consideration for band gap calculation.

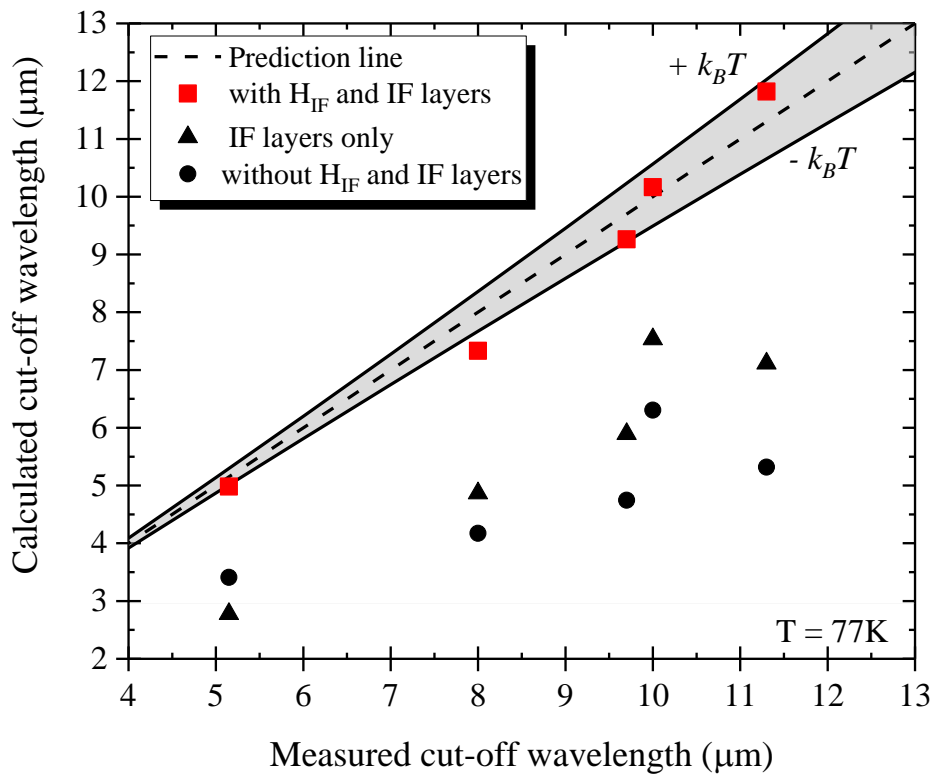

Figure 1: Calculated cut-off wavelength (squares) as a function of the measured cut-off at $77 \mathrm{~K}$ for the different SL samples along with the ideal prediction line (dashed line). The cut-off wavelengths calculated without taking into account the interface matrix (triangles) and without considering either the $H_{I F}$ or the InSb layer at the IFs (circles) are also plotted for comparison. The $\pm k_{B} T$ deviation in the predicted cut-off is represented by the solid lines and the grey area.

After fitting the energy band gap, the electronic band structure ( $E(k)$ dispersion curve) of different SLs has been calculated for different directions of the $\mathrm{k}$ vector. An example of an $E(k)$ plot is represented in Figure 2 for a 12/4 SL for two in-plane directions in the Brillouin zone $k_{/ /}$and in the perpendicular direction $k_{\perp}$. From this figure, the spin-splitting can be seen in the parallel direction as observed in Ref. [11] for non-zero value of the $\alpha$ parameter in Eq. (1). 
Return to the Manage Active Submissions page at http://spie.org/submissions/tasks.aspx and approve or disapprove this submission. Your manuscript will not be published without this approval. Please contact author_help@spie.org with any questions or concerns.

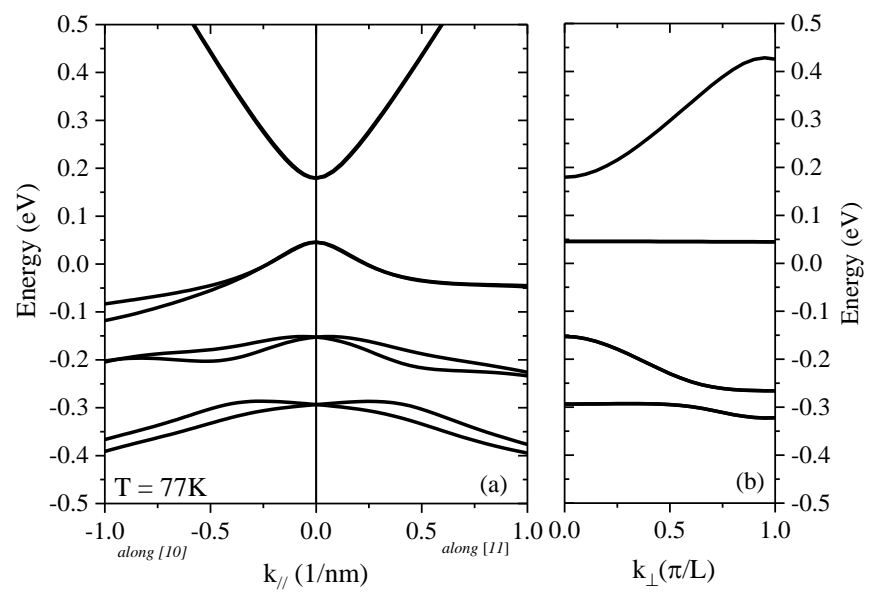

Figure 2: Electronic band structure of a 12/4 SL calculated at $\mathrm{T}=77 \mathrm{~K}$ for (a) two in-plane directions in the Brillouin zone and (b) in the perpendicular direction. In the growth direction the $k$ vector is in units of $\pi / \mathrm{L}$ with $\mathrm{L}$ the period thickness.

\subsection{Electron effective mass}

From Figure 2, it is possible to extract the effective electron mass $m_{e, / /}^{*}$ and $m_{e, \perp}^{*}$ at the band edge (second derivative at the Brillouin zone center) for both the in-plane and perpendicular directions, respectively. $m_{e, / /}^{*}$ and $m_{e, \perp}^{*}$ have been calculated for different InAs/GaSb SL periods as plotted in Figure 3. As a guide for the eyes, different areas corresponding to different values of the ratio $\mathrm{R}$ of the InAs and GaSb layer thicknesses are indicated. $\mathrm{R}>1$ means that the SL period is mainly composed of InAs, while for $\mathrm{R}<1$, it is mainly composed of GaSb. $\mathrm{R}=1$ is for a 'symmetrical' SL for which the thickness of the InAs layer is equal to the thickness of the GaSb layer.

(a) In-plane electron effective mass $\mathrm{m}_{\mathrm{e} / /}^{*}\left[/ \mathrm{m}_{0}\right]$
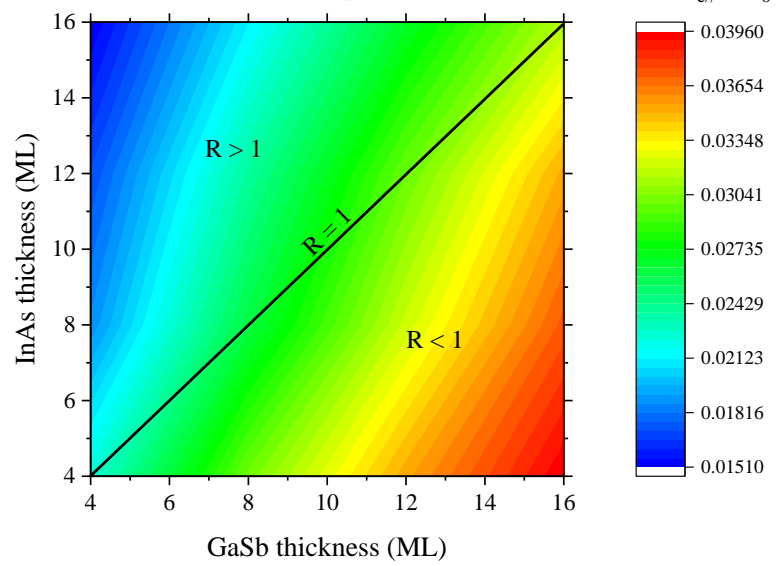

(b) Perpendicular electron effective mass $\mathrm{m}_{\mathrm{e} \perp}^{*}\left[/ \mathrm{m}_{0}\right]$

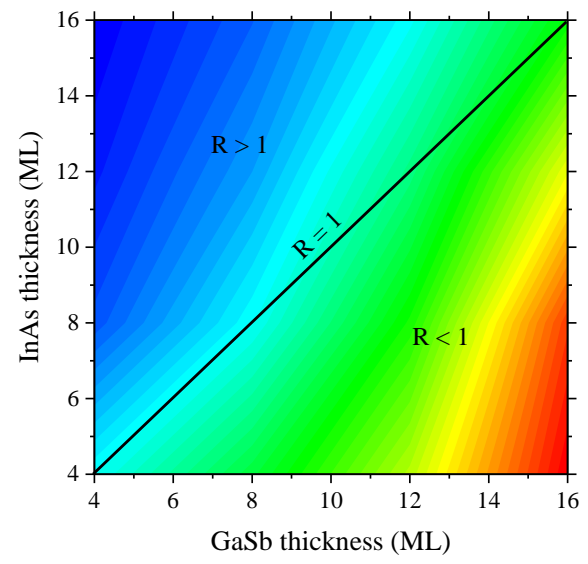

Figure 3: Color map of the (a) in-plane electron effective mass and (b) perpendicular effective mass calculated for as a function of the InAs and GaSb thicknesses. $\mathrm{R}$ is the ratio of the InAs and $\mathrm{GaSb}$ thicknesses within the period $(\mathrm{R}=\mathrm{InAs} / \mathrm{GaSb})$.

For SL periods with layer thicknesses varying from 4 to $16 \mathrm{MLs}, m_{e_{/ /}}^{*}$ is in the range of $(0.0151-0.0396) m_{0}$ (with $m_{0}$ the free electron mass) while the value of $m_{e_{\perp}}^{*}$ is between $(0.022-0.0444) m_{0}$. We can also notice in Figure 3 that whatever the direction the electron effective mass for a SL mainly composed of GaSb $(\mathrm{R}<1)$ is larger than for a SL with $\mathrm{R}>1$. 


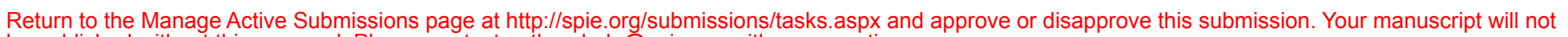
be published without this approval. Please contact author_help@spie.org with any questions or concerns.

The effective electron mass $m_{e}^{*}$ in the InAs/GaSb SL has been calculated as $m_{e}^{*}=m_{e, / /}^{*}{ }^{2 / 3} \cdot m_{e, \perp}^{*}{ }^{1 / 3}$ for different SL periods and is plotted in Figure 4 as a function of the energy band gap $E_{g}$ at $77 \mathrm{~K}$. The horizontal solid lines are calculated for a fixed GaSb layer thickness (from 4 to $16 \mathrm{MLs)}$ ) while the vertical symbols represent data calculated for a fixed InAs layer thickness (from 8 to $16 \mathrm{MLs}$ ).

It can be seen from Figure 4 that the electron effective mass depends mainly on the SL period composition and thickness and slightly on the energy band gap which is not the case for bulk materials. In the SL, the effective mass is mainly influenced by the electron wavefunctions overlap between adjacent InAs wells and less by the interaction between the conduction and valence band. For example, $m_{e}^{*}$ for the $8 / 6 \mathrm{SL}$ with an energy band gap of $245 \mathrm{meV}$ is equal to $0.0235 m_{0}$ whereas for the $16 / 6 \mathrm{SL}$ with $\mathrm{E}_{\mathrm{g}}$ of $88 \mathrm{meV}, m_{e}^{*}$ is $0.0196 m_{0}$. However, for 12/4 SL and 14/12 SL, which have a similar $\mathrm{E}_{\mathrm{g}} \sim 135 \mathrm{meV}$, the electron effective mass is $0.0188 m_{0}$ and $0.0284 m_{0}$, respectively. The calculated value of $m_{e}^{*}$ in the range of $(0.015$ to 0.040$) m_{0}$ for the SL periods studied here. In comparison, the electron effective mass in the HgCdTe (MCT) material is varying with the energy band gap as $0.071 \times E_{g}$ [24] which is smaller than in InAs/GaSb SLs as illustrated in Figure 4. With the tunneling probability being exponentially dependent on the effective mass, the tunneling contribution in SLs is thus lower than in $\mathrm{HgCdTe}$, especially in the LWIR range where the effective mass in $\mathrm{HgCdTe}$ is really small compared to the one in SL.

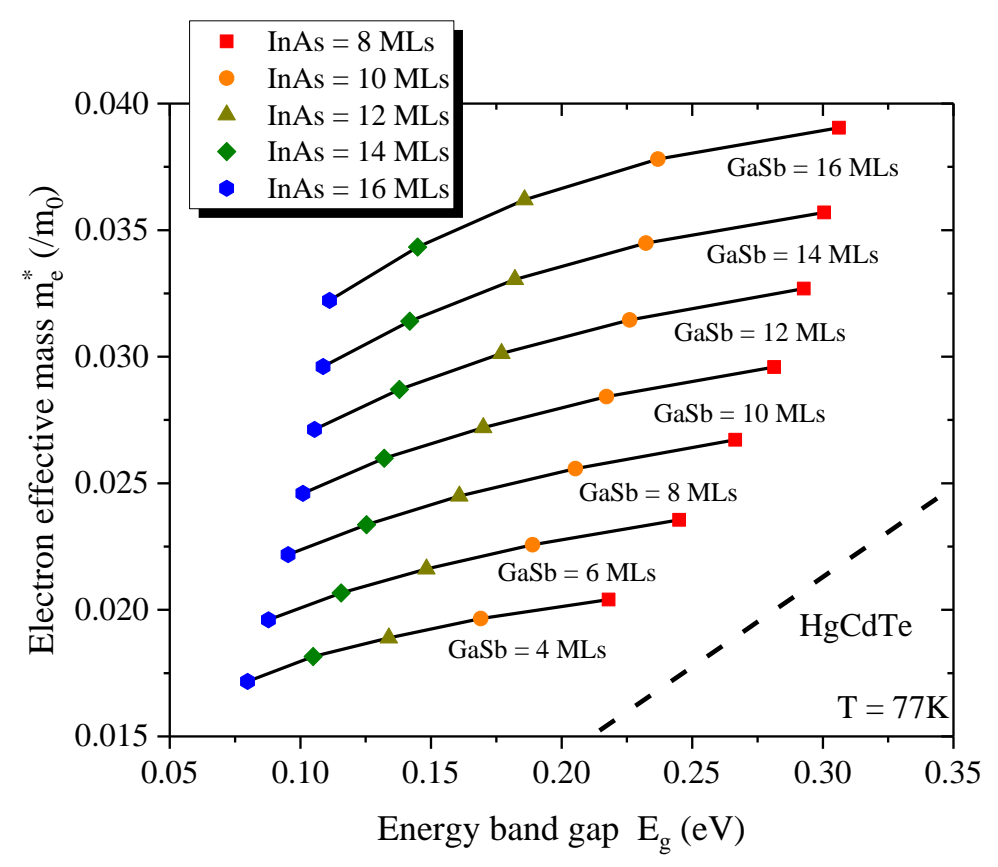

Figure 4: Effective electron mass as a function of the energy band gap at 77K, calculated for different InAs and GaSb layer thicknesses. The horizontal solid lines are calculated for a fixed GaSb layer thickness (indicated below). From bottom to top the GaSb thickness increases from 4 to $16 \mathrm{MLs}$. The same vertical symbols represent data calculated for a fixed InAs layer thickness. From right to left, the InAs thickness increases from 8 to 16 MLs. For comparison, the effective electron mass in $\mathrm{HgCdTe}$ material is also represented.

In addition, Figure 4 illustrates the great tunability that the InAs/GaSb SL material offers. The same energy band gap can be addressed by different SL periods which have different electronic band structure, and therefore different intrinsic properties. The period flexibility has already been experimentally investigated in the MWIR spectral range, and the influence of the period design on the electro-optical properties of pin photodiodes demonstrated [25-26]. In the following section, two different SL periods, having a cut-off wavelength around $10 \mu \mathrm{m}$, are studied. The electronic band structure is first discussed and the influence of the SL design on the electrical properties of a $n B p$ structure is then theoretically evaluated. 
Return to the Manage Active Submissions page at http://spie.org/submissions/tasks.aspx and approve or disapprove this submission. Your manuscript will not be published without this approval. Please contact author_help@spie.org with any questions or concerns.

\subsection{Influence of the InAs/GaSb SL design on the band structure and the electrical characteristics of $n B p$ structure for LWIR detection}

When properly designed, the $n B p$ structure is diffusion-limited at any given temperature. The diffusion current depends on the diffusion length of the minority carrier and the intrinsic carrier concentration $n_{i}=\sqrt{N_{c} \cdot N_{v}} \exp \left(-E_{g} / 2 k_{B} T\right)$ with $N_{c}$ and $N_{v}$ the effective density of states in the conduction and valence band, respectively, and it is inversely proportional to the product $\tau \times N$ with $\tau$ the minority carrier lifetime and $N$ the doping concentration in the absorber layer. By maximizing $\tau \times N$, the diffusion contribution can be minimized. However, the optimal $\tau \times N$ value is ruled by the Auger recombination process which limits the lifetime at a certain doping concentration, around $1 \times 10^{17} \mathrm{~cm}^{-3}$ for LWIR InAs/GaSb SLs [2].

The objective of this section is to compare theoretically the 14/7 SL $(\mathrm{R}=2)$ - used by many research groups for LWIR detection [4-5][27-30] - with a $12 / 2 \mathrm{SL}(\mathrm{R}=6)$ which has a similar band gap. The calculated $E(k)$ dispersion plot for different directions of the $k$ vector is represented in Figure 5 for both SL designs. The energy band gap at $77 \mathrm{~K}$ is 0.122 and $0.118 \mathrm{eV}$ for the 14/7 SL and 12/2 SL, respectively.

Although both SLs have a similar band gap, their electronic band structure presents some clear differences. Firstly, we can see that the valence bands are pushed down for the thinner SL. In particular, the lower ones are further removed from the top valence band (corresponding to the heavy hole). Using a 12/2 SL one could further minimize/suppress Auger recombination in p-type SLs enhancing carrier lifetime (at high doping concentration) [31]. In addition, the spin-splitting observed in the in-plane direction is smaller for the SL with thinner layers because of the delocalization of the hole wavefunction. This delocalization of carriers is also noticeable on the value of the wavefunction overlap of the first heavy hole and first electron quantized states $\left(\left|<f_{e 1}\right| f_{h 1}>\left.\right|^{2}\right)$ which has been determined to be $40 \%$ and $74 \%$ for the $14 / 7 \mathrm{SL}$ and $12 / 2 \mathrm{SL}$, respectively. Such an increase of the wavefunction overlap can result in an enhancement of the absorption coefficient, and thus of the external quantum efficiency of the device. Furthermore, the electron and heavy hole effective masses are larger in the 14/7 SL, in particular the hole effective mass in the growth direction (Table 3). This can be seen on the $E(k)$ plot where the bands tend to be "flatter" for the 14/7 SL than for the $12 / 2 \mathrm{SL}$. Although a larger effective mass is required to reduce the tunneling contribution, in the case of a $n B p$ structure it will mainly impact on the diffusion component via the intrinsic carrier concentration. To evaluate and quantify this impact, the dark-current of a $n B p$ structure with the 14/7 SL and the 12/2 SL has been calculated. Note that the material parameters of both SL designs along with the method to determine them are reported in Table 3.
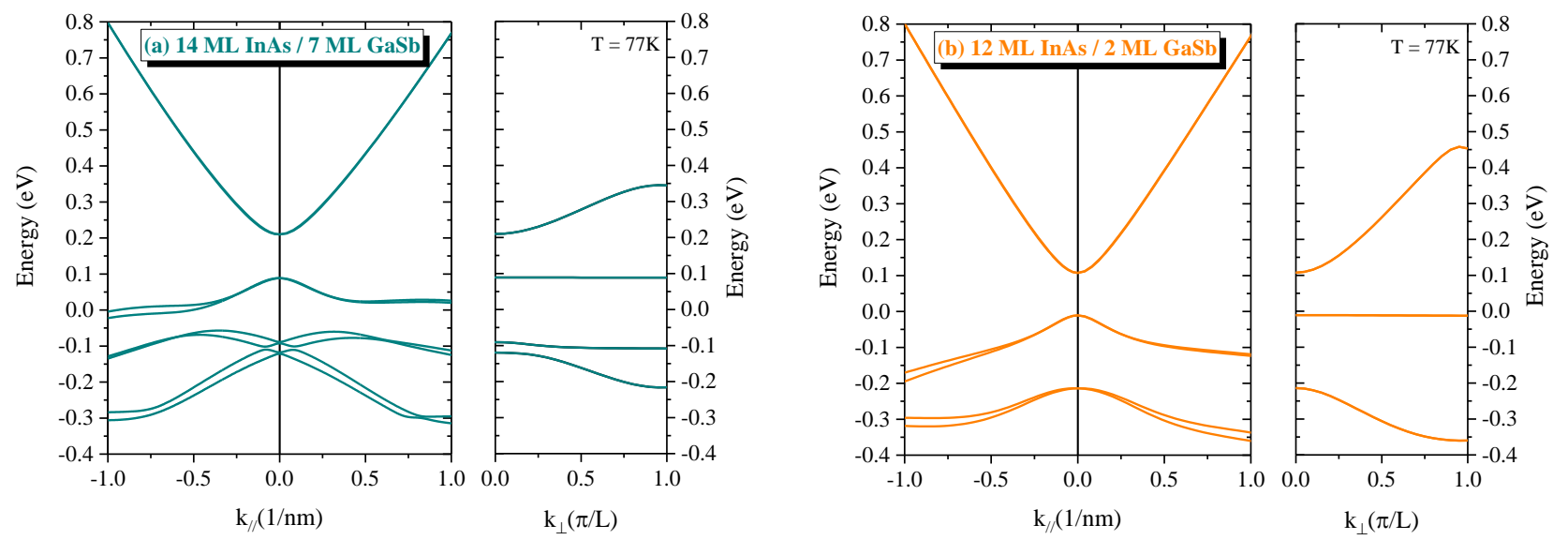

Figure 5: Calculated band structure at a temperature of 77K for different directions of the $k$ vector for the (a) 14/7 SL and (b) $12 / 2 \mathrm{SL}$.

In the following, we assume that the minority carrier lifetime is equal to $30 \mathrm{~ns}$ whatever the SL design [32] and the doping concentration level of the p-type absorber is $1 \times 10^{16} \mathrm{~cm}^{-3}$. We consider that the diffusion length is not the limiting factor of our device, i.e. it is larger than the absorber thickness of $4 \mu \mathrm{m}$ [33-34]. Therefore, the only difference between both structures comes from the intrinsic carrier concentration. Although, in Ref. [34] it is mentioned that the intrinsic carrier 
Return to the Manage Active Submissions page at http://spie.org/submissions/tasks.aspx and approve or disapprove this submission. Your manuscript will not be published without this approval. Please contact author_help@spie.org with any questions or concerns.

concentration is almost independent of the SL period, we observed a slight difference between the 14/7 SL and 12/2 SL. This may come from the fact that the method to determine the effective mass from the electronic band structure is different in Ref. [34]. In addition, the ratio of the InAs and GaSb layer thicknesses is ranging from 1.4 to 2.5 whereas in our case the difference of ratio between the 14/7 SL and 12/2 SL is quite large (factor of 3).

Table 3: Material parameters of the 14/7 SL and 12/2 SL. The method to determine these parameters is also indicated.

\begin{tabular}{|c|c|c|c|}
\hline Parameters & $\begin{array}{l}\text { 14/7 MLs } \\
\text { InAs/GaSb }\end{array}$ & $\begin{array}{l}\text { 12/2 MLs } \\
\text { InAs/GaSb }\end{array}$ & Method \\
\hline Energy band gap at $\mathrm{T}=77 \mathrm{~K}[\mathrm{eV}]$ & 0.122 & 0.118 & $E(k)$ \\
\hline Wavefunction overlap [\%] & 40 & 70 & $\left|<f_{e 1}\right| f_{h 1}>\left.\right|^{2}$ \\
\hline $\begin{array}{l}\text { Electron effective mass }\left[/ \mathrm{m}_{0}\right] \\
m_{e}^{*} \\
m_{e, / /}^{*} \\
m_{e, \perp}^{*}\end{array}$ & $\begin{array}{l}0.022 \\
0.021 \\
0.024\end{array}$ & $\begin{array}{l}0.016 \\
0.014 \\
0.021\end{array}$ & $\begin{array}{c}m_{e}^{*}=m_{e, / /}^{*}{ }^{2 / 3} \cdot m_{e, \perp}^{*} 1 / 3 \\
E(k) \\
E(k)\end{array}$ \\
\hline $\begin{array}{l}\text { Hole effective mass }\left[/ \mathrm{m}_{0}\right] \\
m_{h}^{*} \\
m_{h, / /}^{*} \\
m_{h, \perp}^{*}\end{array}$ & $\begin{array}{l}0.309 \\
0.033 \\
27.10\end{array}$ & $\begin{array}{l}0.169 \\
0.024 \\
8.325\end{array}$ & $\begin{array}{c}m_{h}^{*}=m_{h, / /}^{*}{ }^{2 / 3} \cdot m_{h, \perp}^{*} 1 / 3 \\
E(k) \\
E(k)\end{array}$ \\
\hline $\begin{array}{l}\text { Effective density of states in } \\
\text { conduction band } N_{c} \text { at } 300 \mathrm{~K}\left[\mathrm{~cm}^{-3}\right]\end{array}$ & $8.16 \times 10^{16}$ & $5.06 \times 10^{16}$ & $N_{c}=2.5 \times 10^{19}\left(m_{e}^{*}\right)^{1.5}\left(\frac{T}{300 K}\right)^{1.5}$ \\
\hline $\begin{array}{l}\text { Effective density of states in } \\
\text { valence band } N_{v} \text { at } 300 \mathrm{~K}\left[\mathrm{~cm}^{-3}\right]\end{array}$ & $4.30 \times 10^{18}$ & $1.73 \times 10^{16}$ & $N_{v}=2.5 \times 10^{19}\left(m_{h}^{*}\right)^{1.5}\left(\frac{T}{300 K}\right)^{1.5}$ \\
\hline Permittivity $\varepsilon_{S L}$ & 15.33 & 15.23 & Weighted average \\
\hline Minority carrier lifetime $\tau[\mathrm{ns}]$ & 30 & 30 & Ref. [32] \\
\hline Tunneling mass $m_{t}\left[/ \mathrm{m}_{0}\right]$ & 0.021 & 0.015 & $1 / m_{t}=1 / m_{e}^{*}+1 / m_{h}^{*}$ \\
\hline
\end{tabular}

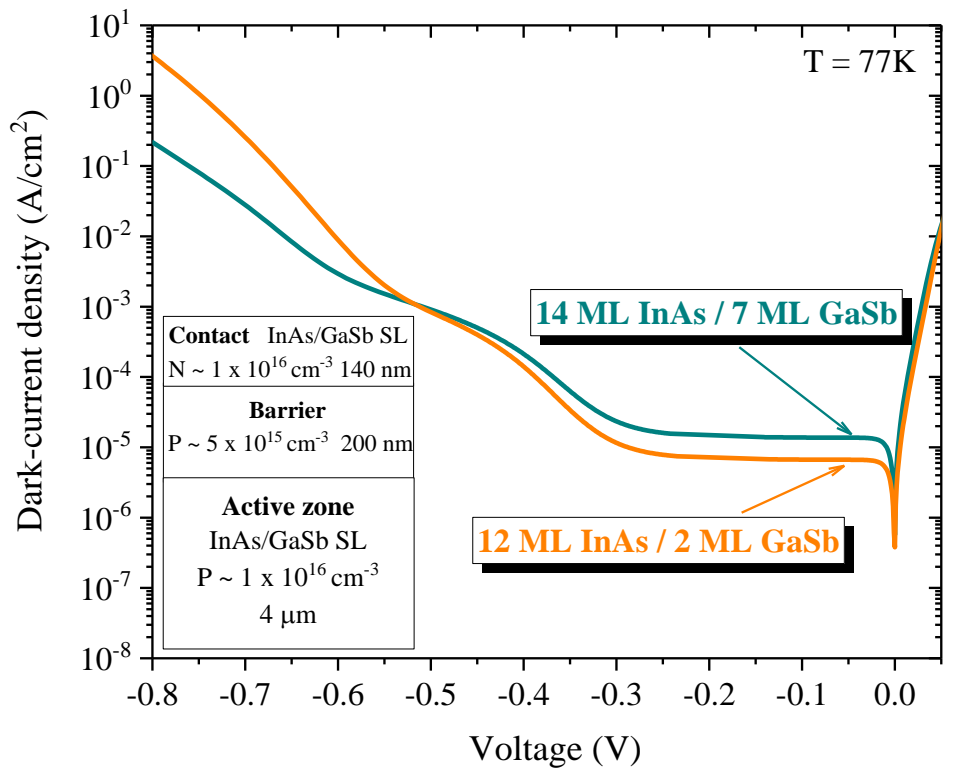

Figure 6: Dark-current density of a $n B p$ device as a function of voltage calculated at $77 \mathrm{~K}$ for the $14 / 7$ SL (blue line) and $12 / 2$ SL (orange line). The $n B p$ barrier structure is presented in inset. 
Return to the Manage Active Submissions page at http://spie.org/submissions/tasks.aspx and approve or disapprove this submission. Your manuscript will not be published without this approval. Please contact author_help@spie.org with any questions or concerns.

The calculated dark-current density of the $n B p$ structure versus voltage at $77 \mathrm{~K}$ is plotted in Figure 6 . The dark-current of both structures has a similar behavior and three regimes can be observed. At high reverse bias $(\mathrm{V}<-0.6 \mathrm{~V})$, the band-toband tunneling contribution is the dominant component of the dark-current, and as already mentioned, it is higher for the $12 / 2 \mathrm{SL}$ due to a smaller effective mass. For $-0.6 \mathrm{~V}<\mathrm{V}<-0.3 \mathrm{~V}$, the generation-recombination current, which includes the trap assisted tunneling current, is the main limiting mechanism. At low reverse bias $(-0.3 \mathrm{~V}<\mathrm{V}<0 \mathrm{~V})$, as expected for a $n B p$ structure the dark-current is diffusion-limited. At $-50 \mathrm{mV}$, it is equal to $1.4 \times 10^{-5}$ and $6.7 \times 10^{-6} \mathrm{~A} / \mathrm{cm}^{2}$ for the 14/7 SL and 12/2 SL, respectively. Although this improvement appears to be less than one order of magnitude on the darkcurrent with the 12/2 SL, the two SL periods have a slight difference in the energy band gap which has a non-negligible effect on the dark-current. For a fair comparison, the dark-current is compared to the well-known MCT Rule07 benchmark at the same cut-off wavelength instead [35]. The dark-current of the 14/7 SL $n B p$ is higher than the Rule07 by a factor of over 3 whereas it is lower by a factor of 0.77 for the $12 / 2 \mathrm{SL} n B p$. These results thus demonstrate that by using the flexibility of the SL design the performances of $n B p$ barrier structure can be improved.

\section{SUMMARY}

In conclusion, an 8-band k.p envelope function method has been used to calculate the electronic band structure of InAs/GaSb superlattices. This model can predict the measured energy band gap of different SLs having different period composition and thickness $(7 / 4,10 / 4,12 / 4,14 / 4$ and 14/7 SLs) within an error corresponding to the thermal broadening in the MWIR and LWIR spectral range. The electron effective mass has been found to be in the range of $(0.015$ to 0.040$) m_{0}$ which is larger than for $\mathrm{HgCdTe}$ material. Finally, the conventional 14/7 SL and a 12/2 SL having a cut-off wavelength close to $10 \mu \mathrm{m}$ have been compared. First, the electron-hole wavefunction overlap of the 12/2 SL has been estimated to be $\sim 74 \%$ which is almost twice the value calculated for a 14/7 SL ( 40\%). This can result in an enhancement of the absorption coefficient for the thinner period thanks to the delocalization of carriers, which also leads to smaller carrier effective masses in the 12/2 SL. This difference has an influence on the intrinsic carrier concentration and therefore on the electrical characteristic of $n B p$ structure. The dark-current of the 14/7 SL $n B p$ is higher than the Rule07 benchamark by a factor of over 3, whereas for the $12 / 2 \mathrm{SL} n B p$ it is lower by a factor of 0.77 , demonstrating that the SL design can be used to improve the overall device performances.

\section{ACKNOWLEDGMENT}

This project has received funding from the European Union's Horizon 2020 research and innovation programme under the Marie Sklodowska-Curie grant agreement No 743521 (Project acronym: ASISA). The authors would also like to acknowledge the financial support provided by Sêr Cymru National Research Network in Advanced Engineering and Materials and by the EPSRC for the Future Compound Semiconductor Manufacturing Hub, reference number EP/P006973/1.

\section{REFERENCES}

[1] D.L. Smith and C. Mailhiot, "Proposal for strained type II superlattice infrared detectors", Journal of Applied Physics 62, 2545 (1987).

[2] A. Rogalski, M. Kopytko, P. Martyniuk, "InAs/GaSb type-II superlattice infrared detectors: three decades of development", Proceedings of SPIE 10177, Infrared Technology and Applications XLIII, 1017715-1 (2017).

[3] P. Manurkar, S. Ramezani-Darvish, B-M Nguyen, M. Razeghi, J. Hubbs, "High performance long wavelength infrared mega-pixel focal plane array based on Type-II superlattices", Applied Physics Letters 97, 193505 (2010).

[4] A. D. Hood, A. J. Evans, A. Lkhlassi, D. L. Lee and W. E. Tennant, "LWIR Strained-Layer Superlattice Materials and Devices at Teledyne Imaging Sensors", Journal of Electronic Materials 39, 1001-1006 (2010).

[5] P.C. Klipstein, E. Avnon, Y. Benny, R. Fraenkel, A. Glozman, S. Grossman, O. Klin, L. Langoff, Y. Livneh, I. Lukomsky, M. Nitzani, L. Shkedy, I. Shtrichman, N. Snapi, A. Tuito, E. Weiss, "InAs/GaSb Type II superlattice barrier devices with a low dark current and high quantum efficiency", Proceedings of SPIE 9070, Infrared Technology and Applications XL, 90700U-1. (2014). 
Return to the Manage Active Submissions page at http://spie.org/submissions/tasks.aspx and approve or disapprove this submission. Your manuscript will not be published without this approval. Please contact author_help@spie.org with any questions or concerns.

[6] L. Höglund, J. B. Rodriguez, R. Marcks von Würtemberg, S. Naureen, R. Ivanov, C. Asplund, R. Alchaar, P. Christol, A. Rouvié, J. Brocal, O. Saint-Pé, E. Costard, "Influence of shallow versus deep etching on dark current and quantum efficiency in InAs/GaSb superlattice photodetectors and focal plane arrays for long wavelength infrared detection", Infrared Physics and Technology 95, 158-163 (2018).

[7] https://www.nextnano.de/nextnano3/

[8] https://www.silvaco.com/products/tcad/device simulation/atlas/atlas.html

[9] Selected Topics of Semiconductor Physics and Technology (G. Abstreiter, M.-C. Amann, M. Stutzmann, and P. Vogl, eds.), Vol. 135, Verein zur Förderung des Walter Schottky Instituts der Technischen Universität München e.V., München, 239 pp. (2011). ISBN 978-3-941650-35-0.

[10]P. C. Klipstein, "Operator ordering and interface-band mixing in the Kane-like Hamiltonian of lattice-matched semiconductor superlattices with abrupt interfaces", Physical Review B 8, 235314 (2010).

[11]Y. Livneh, P. C. Klipstein, O. Klin, N. Snapi, S. Grossman, A. Glozman, E. Weiss, "k·p model for the energy dispersions and absorption spectra of InAs/GaSb type-II superlattices", Physical Review B 86, 235311 (2012).

[12]R. Kaspi, J. Steinshnider, M. Weimer, C. Moeller, A. Ongstad, "As-soak control of the InAs-on-GaSb interface", Journal of Crystal Growth 225, 544-549 (2001).

[13]E. Plis, S. Annamalai, K. T. Posani, S. Krishna, R. A. Rupani, S. Ghosh, "Midwave infrared type-II InAs/GaSb superlattice detectors with mixed interfaces", Journal of Applied Physics 100, 014510 (2006).

[14]H. J. Haugan, G. J. Brown, L. Grazulis, "Effect of interfacial formation on the properties of very long wavelength infrared InAs/GaSb superlattices", Journal of Vacuum Science \& Technology B 29, 03 C101 (2011).

[15] J. B. Rodriguez, P. Christol, L. Cerutti, F. Chevrier, A. Joullié, "MBE growth and characterization of type-II InAs/GaSb superlattices for mid-infrared detection", Journal of Crystal Growth, 274, 6-13 (2005).

[16]Y. Livneh, P. C. Klipstein, O. Klin, N. Snapi, S. Grossman, A. Glozman, E. Weiss, "Erratum: k·p model for the energy dispersions and absorption spectra of InAs/GaSb type-II superlattices", Physical Review B 90, 039903(E) (2014).

[17]I. Vurgaftman, J. R. Meyer, L. R. Ram-Mohan, "Band parameters for III-V compound semiconductors and their alloys", Journal of Applied Physics 89, 5815-5875 (2001).

[18]E. Halvorsen, Y. Galperin, K. Chao, "Optical transitions in broken gap heterostructures”, Physical Review B 61, $16743-16749$ (2000).

[19]S. H. Wei and A. Zunger, "Predicted band-gap pressure coefficients of all diamond and zinc-blende semiconductors: Chemical trends", Physical Review B 60, 5404-5411 (1999).

[20]K. Banerjee, J. Huang, and S. Ghosh, "Modeling and simulation of long-wave infrared InAs/GaSb strained layer superlattice photodiodes with different passivants", Infrared Physics and Technology 54, 460-464 (2011).

[21] J. Abautret, J. P. Perez, A. Evirgen, F. Martinez, P. Christol et al., "Electrical modeling of InSb PiN photodiode for avalanche operation", Journal of Applied Physics 113, 183716 (2013).

[22] M. Delmas, J.-B. Rodriguez, P. Christol, "Electrical modeling of InAs/GaSb superlattice mid-wavelength infrared pin photodiode to analyze experimental dark current characteristics", Journal of Applied Physics 116, 113101 (2014).

[23] M. Delmas, R. Rossignol, J. B. Rodriguez, P. Christol, "Design of InAs/GaSb superlattice infrared barrier detectors", Superlattices and Microstructures 104, 402-414 (2017).

[24] A. Rogalski, "HgCdTe infrared detector material: history, status and outlook", Reports on Progress in Physics 68, 2267-2336 (2005).

[25]R. Taalat, J. -B. Rodriguez, M. Delmas, P. Christol, "Influence of the period thickness and composition on the electro-optical properties of type-II InAs/GaSb midwave infrared superlattice photodetectors", Journal of Physics D: Applied Physics 47, 015101 (2014).

[26]E. Giard, I. Ribet-Mohamed, J. Jaeck, T. Viale, R. Haïdar, R. Taalat, M. Delmas, J. -B. Rodriguez, E. Steveler, N. Bardou, F. Boulard, P.Christol, "Quantum efficiency investigations of type-II InAs/GaSb midwave infrared superlattice photodetectors", Journal of Applied Physics 116, 1-7 (2014).

[27]D. Z. Y. Ting, C. J. Hill, A. Soibel, S. A. Keo, J.M. Mumolo, J. Nguyen, S. D. Gunapala, "A high-performance long wavelength superlattice complementary barrier infrared detector", Applied Physics Letters 95, 3-5 (2009).

[28]B. -M. Nguyen, D. Hoffmann, P. -Y. Delaunay, E. K. Huang, M. Razeghi, "Very high performance LWIR and VLWIR Type-II InAs/GaSb superlattice photodiodes with M-structure barrier", Proceedings of SPIE 7082, Infrared Spaceborne Remote Sensing and Instrument XVI, 708205 (2008).

[29] N. Gautam, E. Plis, H.S. Kim, M. N. Kutty, S. Myers, A. Khoshakhlagh, L. R. Dawson, S. Krishna, "Heterostructure Band Engineering of Type-II InAs/GaSb superlattice based Longwave Infrared Photodiodes 
Return to the Manage Active Submissions page at http://spie.org/submissions/tasks.aspx and approve or disapprove this submission. Your manuscript will not be published without this approval. Please contact author_help@spie.org with any questions or concerns.

using Unipolar Current Blocking Barriers", Proceedings of SPIE 7660, Infrared Technology and Applications XXXVI, 76601T-1 (2010).

[30] X. Han, D. Jiang, G. Wang, H. Hao, Y. Sun, Z. Jiang, Y. Lv, C. Guo, Y. Xu, Z. Niu, "Small-pixel long wavelength infrared focal plane arrays based on InAs/GaSb Type-II superlattice", Infrared Physics and Technology 89, 3540 (2018).

[31]C. H. Grein, W. H. Lau, T. L. Harbert, M. E. Flatté, "Modeling of very long infrared wavelength InAs/GaInSb strained layer superlattice detectors", Materials for Infrared Detectors II, Proceedings of SPIE 4795, 39-43 (2002).

[32]D. Donetsky, G. Belenky, S. Svensson, S. Suchalkin, "Minority carrier lifetime in type-2 InAs-GaSb strainedlayer superlattices and bulk HgCdTe materials", Applied Physics Letters, 97, 36-38 (2010).

[33] L. Höglund, J. B. Rodriguez, S. Naureen, R. Ivanov, C. Asplund, R. Marcks von Würtemberg, R. Rossignol, P. Christol, A. Rouvié, J. Brocal, O. Saint-Pé, E. Costard, "Very long wavelength type-II InAs/GaSb superlattice infrared detectors", Proceedings of SPIE 10624, Infrared Technology and Applications XLIV, 106240I (2018).

[34]P. C. Klipstein, Y. Benny, S. Gliksman, A. Glozman, E. Hojman, O. Klin, L. Langof, I. Lukomsky, I. Marderfeld, M. Nitzani, N. Snapi, E. Weiss, "Minority carrier lifetime and diffusion length in type II superlattice barrier devices", Infrared Physics and Technology 96, 155-162 (2019).

[35] W. E. Tennant, "Rule 07"' revisited: Still a good heuristic predictor of p/n HgCdTe photodiode performance?", Journal of Electronic Materials, 39, 1030-1035 (2010). 